\title{
BMJ
}

\section{Effect of $50000 \mathrm{IU}$ vitamin A given with BCG vaccine on mortality in infants in Guinea-Bissau: randomised placebo controlled trial}

\author{
Christine Stabell Benn, senior researcher, ${ }^{1}$ Birgitte Rode Diness, clinician, ${ }^{1}$ Adam Roth, clinician, ${ }^{1,2}$ Ernesto \\ Nante, clinician, ${ }^{3}$ Ane Bærent Fisker, student, ${ }^{3}$ Ida Maria Lisse, senior registar , ${ }^{4}$ Maria Yazdanbakhsh, \\ professor , ${ }^{5}$ Hilton Whittle, professor , ${ }^{6}$ Amabelia Rodrigues, research director, ${ }^{3}$ Peter Aaby, director ${ }^{3}$
}

\section{Bandim Health Project, Statens Serum Institut, Artillerivej 5, 2300 Copenhagen S, Denmark \\ ${ }^{2}$ Department of Medical Microbiology, Lund University, 20502 Malmö, Sweden \\ ${ }^{3}$ Bandim Health Project, Indepth Network, Apartado 861, Bissau, Guinea-Bissau \\ ${ }^{4}$ Department of Pathology, Herlev University Hospital, 2730 Herlev, Denmark \\ ${ }^{5}$ Department of \\ Immunoparasitology, Leiden \\ University Medical Centre, Leiden 2333 AZ, Netherlands}

${ }^{6}$ MRC Laboratories, Fajara, POB 273, Gambia

Correspondence to: C S Benn cb@ssi.dk

doi:10.1136/bmi.39542.509444.AE

\section{ABSTRACT}

Objective To investigate the effect of high dose vitamin A supplementation given with BCG vaccine at birth in an African setting with high infant mortality.

Design Randomised placebo controlled trial.

Setting Bandim Health Project's demographic surveillance system in Guinea-Bissau, covering approximately 90000 inhabitants.

Participants 4345 infants due to receive BCG. Intervention Infants were randomised to 50000 IU vitamin A or placebo and followed until age 12 months.

Main outcome measure Mortality rate ratios.

Results 174 children died during follow-up (mortality= 47/1000 person-years). Vitamin A supplementation was not significantly associated with mortality; the mortality rate ratio was 1.07 (95\% confidence interval 0.79 to 1.44 ). The effect was 1.00 ( 0.65 to 1.56$)$ during the first four months and 1.13 ( 0.75 to 1.68$)$ from 4 to 12 months of age. The mortality rate ratio in boys was 0.84 ( 0.55 to 1.27$)$ compared with 1.39 ( 0.90 to 2.14 ) in girls ( $P$ for interaction=0.10). An explorative analysis revealed a strong interaction between vitamin $A$ and season of administration.

Conclusions Vitamin A supplementation given with BCG vaccine at birth had no significant benefit in this African setting. Although little doubt exists that vitamin A supplementation reduces mortality in older children, a global recommendation of supplementation for all newborn infants may not contribute to better survival. Trial registration Clinical trials NCT00168597.

\section{INTRODUCTION}

High dose vitamin A supplementation in children aged above 6 months is associated with significant reductions in overall mortality. ${ }^{1}$ In contrast, vitamin A supplementation between 1 month and 5 months of age has not been associated with beneficial effects on mortality. ${ }^{2}$ However, when we started our study, two trials from Indonesia and India had both found a significantly beneficial effect of vitamin A supplementation at birth; the reduction in mortality was $64 \%$ in Indonesia and 21\% in India. ${ }^{34}$ In both trials the effect was strongest for boys, with a significant $85 \%$ reduction in mortality for boys and only a non-significant $16 \%$ reduction for girls in Indonesia and a significant 30\% reduction in boys compared with a non-significant $13 \%$ reduction in girls in India. The studies followed the infants to 12 months of age in Indonesia and 6 months in India, and both saw the beneficial effect during the first three to four months of life. ${ }^{34}$ In Indonesia, the effect was significantly better among normal birthweight infants than in low birthweight infants, ${ }^{3}$ whereas the opposite was noted in India. ${ }^{4}$

Because of the intriguing age pattern of the effect of vitamin A supplementation on mortality, we hypothesised that vitamin A supplementation interacted with routine childhood vaccinations and was beneficial when given with the live BCG vaccine at birth or live measles vaccine at 6-9 months of age but not when given with inactivated diphtheria-tetanus-pertussis vaccine at 1-5 months of age. ${ }^{2}$ This hypothesis could explain the lack of effect of vitamin A supplementation in low birthweight infants in Indonesia, as BCG was postponed for low birthweight infants. In India, the BCG coverage was low, but no special policy existed for low birthweight infants.

We aimed to investigate the effect of high dose vitamin A supplementation given with BCG vaccine at birth in an African setting with high infant mortality. As BCG vaccine is postponed for low birthweight infants weighing below $2500 \mathrm{~g}$, we enrolled only infants weighing $2500 \mathrm{~g}$ or more. The primary study hypothesis was that vitamin A supplementation would be associated with at least a $30 \%$ reduction in mortality during the first year of life. We expected the effect to be most pronounced within the first four months of life as in Asia. Furthermore, evidence for sex differential effects of vitamin A supplementation accumulating during the trial made us hypothesise that supplementation would be particularly beneficial for boys. ${ }^{5-7}$

\section{METHODS}

Setting

The Bandim Health Project has a demographic surveillance system in six suburban districts of the 
capital of Guinea-Bissau and covers approximately 90000 inhabitants. The study area includes three health centres, and a national hospital is a few kilometres away. The Bandim Health Project has assistants placed at the health centres and the hospital to register all children in the study area. All houses in the study area are visited monthly to register new pregnancies and births. Once a newborn infant is identified, the child is followed with home visits every third month to register vaccinations, infections, hospital admissions, feeding patterns, and survival.

Guinea-Bissau follows the World Health Organization's recommendations of providing BCG and oral polio vaccine at birth, combined diphtheria-tetanuspertussis and oral polio vaccine at 6,10 , and 14 weeks of age, and measles vaccine at 9 months of age. However, during 2004 Guinea-Bissau lacked oral polio vaccine for several periods. Also, while the trial was ongoing an early measles vaccination trial was started in the study area and some children received measles vaccine at 4 . 5 months of age. By 12 months of age, $90 \%$ of the surviving children had received three diphtheriatetanus-pertussis vaccines and $86 \%$ had received a measles vaccine. None the less, a measles epidemic occurred in the winter of 2003-4. While we were doing the trial, the prevalence of HIV-1 was around 3-5\% in the study area among women of fertile age (sentinel surveillance, unpublished data). A large proportion of pregnant women are screened for HIV to prevent vertical transmission. Uncontrolled, vertical transmission would be around $25 \%{ }^{8}$ With the vertical transmission control programme ongoing, we expect less than $1 \%$ of the children to be infected with HIV-1. All patients with tuberculosis in the area are registered and offered treatment. The WHO's recommendations for vitamin A supplementation after 6 months of age are not implemented in Guinea-Bissau. Almost all children in the study area are breast fed to at least 18 months of age. While this study was ongoing, two national vitamin A campaigns took place in November 2003 and November 2004, during which children aged between 6 months and 5 years were offered vitamin A supplementation. The Bandim Health Project registered all the children in the study area who received vitamin A supplementation during these campaigns.

\section{Local vitamin A status}

Guinea-Bissau is classified by Unicef as having subclinical vitamin A deficiency. ${ }^{9}$ In this trial, we collected blood samples from a subgroup of 614 children at 6 weeks of age and a further blood sample from 369 of these children and their mothers at 4 months of age. ${ }^{10}$ We analysed the samples for retinol binding protein and $\mathrm{C}$ reactive protein. Overall, vitamin A supplementation had no effect on retinol binding protein concentrations at 6 weeks and 4 months of age. Controlled for $\mathrm{C}$ reactive protein, $27 \%$ of the children had retinol binding protein corresponding to values below $0.7 \mu \mathrm{mol} / \mathrm{l}$ retinol (that is, low retinol binding protein) at 6 weeks of age and $9 \%$ had low retinol binding protein at 4 months of age. One mother had low retinol binding protein. Retinol binding protein was higher in boys than in girls at 4 months of age. Furthermore, at 6 weeks of age retinol binding protein was higher among infants enrolled during the dry season than in infants enrolled in the rainy season. ${ }^{10}$

We started the trial procedures in the period from 2 August to 12 November 2002. During this period, we randomised 570 children to 25000 IU vitamin A or placebo. A medical doctor followed all children with home visits on days one, two, and three, and a field assistant subsequently visited the children weekly for a month to detect possible adverse events in the form of bulging fontanelles, vomiting, irritability, infections, fever, skin problems, and healthcare contacts. We found no indications of adverse events, and we proceeded to the next phase of the trial.

\section{Participants}

We invited mothers who gave birth at the maternity wards of the national hospital and a local health centre (Bandim Health Centre) to participate in the study when their child was to receive BCG vaccination after delivery. Furthermore, we invited mothers who delivered at home to participate when they came for $\mathrm{BCG}$ vaccination at two of the three health centres in the study area (Bandim and Belem). We did not include the third health centre (Cuntum) for logistical reasons. The inclusion criteria were weight at least $2500 \mathrm{~g}$ at presentation and no signs of overt illness or malformations. Infants who died in the maternity wards, before the vaccination team arrived in the morning, could not be assessed for enrolment. This was also the case for those born at home who died before their mother brought them for vaccination.

\section{Ethical considerations}

A trained field worker informed mothers about the trial and asked them if they wanted their child to participate. They were informed that all infants would be visited by our team and that all children would receive a vitamin A supplement at age 12 months. They were also informed that they could come to a clinic at the health centre, attended by staff from the Bandim Health Project, for free consultations and essential drugs if their child became ill. The mothers gave oral consent. Those who knew how to write their own name gave written consent; for the others the assistant signed a statement that they had understood the information given and agreed to participate.

\section{Randomisation}

All infants were vaccinated intradermally in the upper left deltoid region with $0.05 \mathrm{ml}$ BCG vaccine (Statens Serum Institut, Copenhagen, Denmark). The mother drew a lot from an envelope prepared by the study supervisor. Each envelope contained 100 lots - 50 marked " 1 " and 50 marked " 2 "- indicating from which of two numbered bottles, " 1 " or " 2 ," the child should receive the supplement. The lots were folded, making it impossible to tell what was written on them before they 
were opened. A new envelope was not taken into use before the previous envelope had been completely emptied. The result of the randomisation was noted on the inclusion form and the lot was stapled to the inclusion form.

At each of the three inclusion sites, one carefully trained assistant carried out the randomisation procedure every day except during short vacations. The dark glass bottles were prepared at Skanderborg Pharmacy, Denmark, and contained 20 doses of $0.5 \mathrm{ml}$ of vegetable oil. Half of the bottles contained $50000 \mathrm{IU}$ vitamin A as retinyl palmitate and $10 \mathrm{IU}$ vitamin $\mathrm{E}$ per $0.5 \mathrm{ml}$ oil; the other half contained only $10 \mathrm{IU}$ vitamin E per $0.5 \mathrm{ml}$ oil. The bottles were kept at $5^{\circ} \mathrm{C}$. A fresh lot was prepared at least once a year. Under such circumstances the stability is very high. ${ }^{11}$ The code was kept at the pharmacy until 12 months after the last child was included.

\section{Intervention}

In accordance with the result of the randomisation procedure, a trained nurse slowly released $0.5 \mathrm{ml}$ oil from either bottle 1 or bottle 2 (that is, either $50000 \mathrm{IU}$ vitamin A or placebo) into the mouth of the child with a sterile syringe.

\section{Blinding}

Apart from the randomisation number, the bottles looked alike; we judged small differences in taste and colour of the contents as unimportant owing to the recipients' age. When asked, none of the three assistants who were responsible for the randomisation procedures at the hospital and at the heath centres had any idea which bottles contained vitamin A and which placebo. We concluded that the blinding of mothers and assistants was successful.

\section{Outcomes examined in the trial}

As reported elsewhere, we followed more than 2000 of the children for adverse events (bulging fontanelles, vomiting, irritability, infections, fever, skin problems, and healthcare contacts) during the first month after supplementation with 50000 IU vitamin A or placebo. ${ }^{12}$ Furthermore, we examined 2710 children for BCG scar formation and delayed type hypersensitivity to purified protein derivative of Mycobacterium tuberculosis at 2 and 6 months of age. ${ }^{13}$ We measured the ex vivo cytokine response to purified protein derivative in 607 infants at age 6 weeks. We concluded that vitamin A supplementation with BCG vaccination was not associated with serious adverse events and did not seem to interfere with the long term immune response to BCG. ${ }^{1213}$

All children were followed through the routine registration system every three months and were visited by a special team at age 12 months. The assistants of the registration system and the special team were unaware of the allocated treatment, " 1 " or " 2 ," as they were not present during enrolment and the information was not transferred to the children's vaccination card or followup forms. Deaths were registered at each visit and

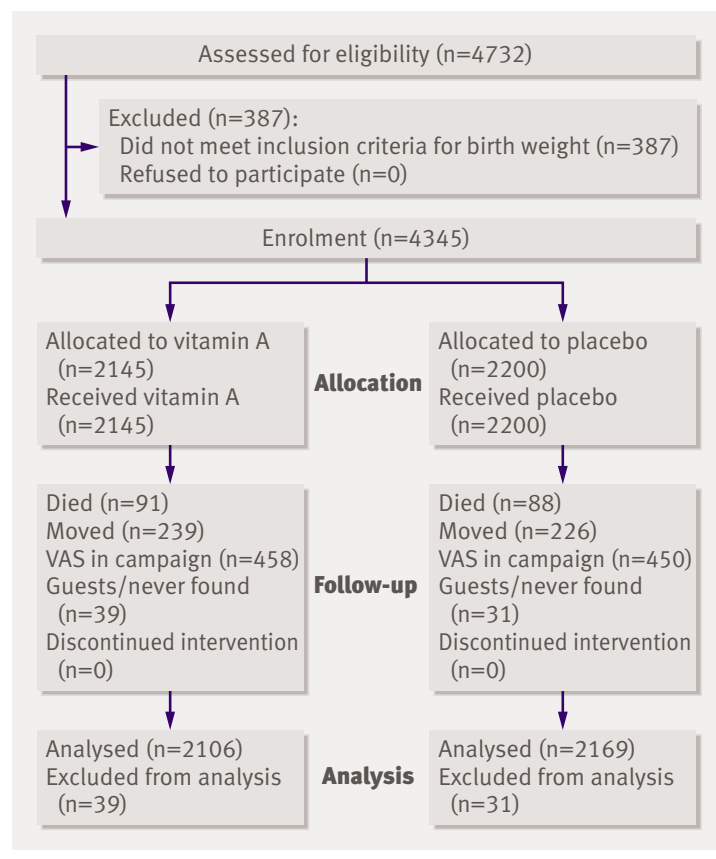

Fig 1 | Trial profile. VAS=vitamin A supplementation

followed by a verbal autopsy by a trained local physician. The physician used a questionnaire designed by the Indepth Network. ${ }^{14}$ Preferably, he interviewed the mother $(58 \%)$, but if this was not feasible he interviewed a relative. On the basis of the questionnaire, a panel of three medical doctors, including at least one local doctor, all ignorant of the randomisation group, reached consensus on the cause of death. If consensus could not be reached a fourth doctor was consulted. Deaths were categorised on the basis of suggestions from WHO. ${ }^{15}$ No death within the first year was due to accident. Children were considered to have been exposed to tuberculosis if they were born within 6 months before a person from the same multi-family house was diagnosed with tuberculosis. All children were offered 100000 IU vitamin A at the 12 months visit.

\section{Sample size}

Assuming a significance level of 5\% and 80\% power, and with an expected infant mortality between day 2 and 12 months of age of $7 \%$ among normal birthweight infants, we estimated that the study would need 2085 children in each group to detect a $30 \%$ change in mortality associated with vitamin A supplementation.

\section{Statistical analysis}

We assessed survival in Cox proportional hazards models with age as the underlying time. ${ }^{16}$ Infants provided person years at risk from enrolment until they reached 12 months of age, moved, or died. As subsequent administration of vitamin A supplementation would neutralise possible differential effects of the original intervention, infants who had received vitamin A supplementation during a campaign did not contribute with follow-up time after the campaign. Five deaths (three in the vitamin A group, two in the 
Table 1| Baseline characteristics of 4345 infants enrolled in vitamin A trial, November 2002November 2004, Guinea-Bissau. Values are numbers (percentages) unless stated otherwise

\begin{tabular}{|c|c|c|}
\hline Characteristic & Vitamin $A(n=2145)$ & Placebo $(n=2200)$ \\
\hline Boys* & $1075(50)$ & $1125(51)$ \\
\hline \multicolumn{3}{|l|}{ Suburb: } \\
\hline Bandim & $1076(50)$ & $1113(51)$ \\
\hline Mindara/Belem/Cuntum & $1030(48)$ & $1056(48)$ \\
\hline Unknown & $39(2)$ & $31(1)$ \\
\hline \multicolumn{3}{|l|}{ Maternal schooling: } \\
\hline No & $624(29)$ & $638(29)$ \\
\hline Yes & $1290(60)$ & $1361(62)$ \\
\hline Unknown & $231(11)$ & $201(9)$ \\
\hline \multicolumn{3}{|l|}{ Maternal ethnicity: } \\
\hline Pepel & $605(28)$ & $606(28)$ \\
\hline Other & $1458(68)$ & $1536(70)$ \\
\hline Unknown & $82(4)$ & $58(3)$ \\
\hline \multicolumn{3}{|l|}{ Electricity: } \\
\hline No electricity & $1379(64)$ & $1416(64)$ \\
\hline Electricity available & $686(32)$ & $726(33)$ \\
\hline Unknown & $80(4)$ & $58(3)$ \\
\hline Enrolment in rainy season* & $1047(49)$ & $1107(50)$ \\
\hline Enrolment at national hospital ${ }^{\star}$ & $1220(57)$ & $1266(58)$ \\
\hline \multicolumn{3}{|l|}{ Exposed to tuberculosis in home: } \\
\hline Yes & $52(2)$ & $42(2)$ \\
\hline No & 2054 (96) & 2127 (97) \\
\hline Unknown & $39(2)$ & $31(1)$ \\
\hline Median (10-90\% range) age at enrolment (days) & $1(0-23)$ & $1(0-21)$ \\
\hline Mean (SD) maternal MUAC (mm) & $255(31)$ & $254(30)$ \\
\hline Mean (SD) weight $(\mathrm{kg})$ & $3.33(0.70)$ & $3.32(0.67)$ \\
\hline Mean $(\mathrm{SD})$ length $(\mathrm{cm})$ & $50(3)$ & $50(3)$ \\
\hline Mean (SD) MUAC (mm) & $101(11)$ & $100(11)$ \\
\hline Mean (SD) head circumference $(\mathrm{cm})$ & $35(2)$ & $35(2)$ \\
\hline Mean (SD) abdominal circumference $(\mathrm{cm})$ & 31 (3) & $31(3)$ \\
\hline Mean $(\mathrm{SD})$ ponderal index $\left(\mathrm{kg} / \mathrm{m}^{3}\right)$ & $26(4)$ & $26(3)$ \\
\hline
\end{tabular}

MUAC=Mid upper arm circumference.

*Variables with two levels (such as included at national hospital/elsewhere) are presented by one of the levels.

placebo group) occurred after a child had received vitamin A supplementation in a campaign and before the child reached 12 months of age; these deaths were not taken into account in the analyses.

A priori, our intention was to study the effect of vitamin A supplementation on overall mortality. Accumulating evidence for sex differential effects of vitamin A supplementation during the trial made us hypothesise before we started the analyses that supplementation would be particularly beneficial for boys. ${ }^{5-7}$ We also did explorative analyses to study whether baseline characteristics such as anthropometric measures, socioeconomic factors, and season could have modified the response to vitamin A supplementation. We analysed effect modification by investigating the homogeneity of the effect of vitamin A supplementation in the different categories of the suspected modifier with Wald test statistics. When looking at the effect of vitamin A supplementation according to birth weight and other birth characteristics, we restricted the analysis to children enrolled on day 0 and day $1(n=2572)$ for whom information on birth characteristics was available ( $\mathrm{n}=2559-69$, depending on birth characteristic) and divided the data into the lowest quarter and the three highest quarters. We used Schoenfeldt's residuals and visual inspection of Nelson-Aalens estimators to test for proportionality of hazard rates.

\section{RESULTS}

\section{Participant flow}

Figure 1 illustrates the trial profile. Between 13 November 2002 and 28 November 2004 we enrolled 4345 children, of whom 174 died within the first year of life - that is, a mortality of $47 / 1000$ person years. A total of 70 children who were said at enrolment at the main hospital to live in the study area either turned out to be temporary guests who left immediately after delivery $(n=36)$ or could not be identified in the neighbourhood indicated by the mother or in the project register $(n=34)$. These infants did not contribute follow-up time to the survival analysis (fig 1). The 70 children lost to follow-up and those followed up did not differ with regard to sex, age, and baseline anthropometrics.

Although the study was planned to examine the effect of vitamin A supplementation administered simultaneously with BCG at birth, several deviations from this plan occurred. Firstly, a total of 85 children, of whom four died (mortality 54/1000 person years), received vitamin A several days after $B C G$ vaccination, most because they were delivered by caesarean section. As recommended, these children were given BCG shortly after birth, but the mother was not available to give informed consent and the children could be enrolled only several days later. The effect of vitamin A supplementation on mortality at 12 months of age was not different when it was given three or more days after $\mathrm{BCG}$ vaccination $(\mathrm{P}=0.92)$.

Secondly, children delivered at home received $\mathrm{BCG}$ and vitamin A supplementation when the child was brought to a healthcare facility. A total of 1181 (27\%) of the children came after the first week of life. Their median age at enrolment was 16 days (10-90 centiles $=$ 9-64 days). Stratification on time of supplementation revealed no indication that the effect of vitamin A supplementation differed with age at supplementation and vaccination $(\mathrm{P}=0.95)$. Thirdly, some children delivered at home or outside the study area came so late for BCG vaccination that they also received the diphtheria-tetanus-pertussis vaccination recommended at 6 weeks of age at the same time or even before BCG ( $n=191$, nine deaths; mortality 73/1000 person years). We found no significant difference between the effect of vitamin A supplementation if BCG was given with or after diphtheria-tetanuspertussis $(\mathrm{P}=0.62)$. We therefore treated all children the same way in the analyses.

\section{Baseline data}

No differences in baseline characteristics existed between the two intervention groups (table 1). We did all analyses without control for these characteristics. 


\section{Outcomes and estimation}

Vitamin A supplementation was not significantly associated with survival during the first year of life; the overall mortality rate ratio was $1.07(95 \%$ confidence interval 0.79 to $1.44 ; \mathrm{P}=0.66$ for effect of vitamin A supplementation) (fig 2). The age at vaccination did not matter for the estimated effect of vitamin A supplementation (data available on request). The inclusion criteria were no malformation and no overt illness, but mortality within the first 7 days of life was none the less high; 20 (mortality $349 / 1000$ person years) of the children died during this period-10 vitamin A ( 6 boys, 4 girls), 10 placebo ( 5 boys, 5 girls), mostly from septicaemia/asphyxia $(n=13)$ or acute haemorrhage $(n=4)$. Excluding the 20 perinatal deaths resulted in an overall effect of vitamin A supplementation of 1.08 (0.79 to 1.48). The previous studies saw a beneficial effect only during the first four months of life. ${ }^{34} \mathrm{We}$ found the mortality rate ratio to be $1.00(0.65$ to 1.56$)$ during this period (79 deaths) and 1.13 (0.75 to 1.68) from 4 to 12 months of age ( 95 deaths).

We censored follow-up time for children when they received vitamin A supplementation in campaigns to avoid dilution of the intervention effects. Without censoring, the estimate was almost unchanged: 1.08 (0.80 to 1.44$)$.

\section{Sex}

We found a tendency for a sex differential effect of vitamin A supplementation; the mortality rate ratio in boys was 0.84 (0.55 to 1.27 ) compared with 1.39 (0.90 to 2.14) in girls ( $\mathrm{P}$ for interaction $=0.10)$ (fig 3). As can also be seen in figure 3 , the differential effect was not apparent before 4 months of age (boys 0.94 ( 0.52 to $1.71)$, girls 1.09 (0.56 to 2.11$)$; P for interaction=0.76) but was marked in the period from 4 to 12 months of age $(0.74$ (0.41 to 1.34 ) versus 1.67 (0.94 to 2.97$)$; $P$ for interaction $=0.05)$.

Explorative analyses

Stratified analyses according to mother/child characteristics

We looked for any differential effect of vitamin A stratified according to characteristics of mothers or children that might be associated with vitamin A status.

\begin{tabular}{|c|c|c|c|}
\hline & \multicolumn{3}{|c|}{ Mortality/1000 person years (deaths/person years) } \\
\hline & All & Boys & Girls \\
\hline \multicolumn{4}{|l|}{ Rainy season } \\
\hline Vitamin A + BCG & $59(54 / 913)$ & $57(26 / 455)$ & $61(28 / 458)$ \\
\hline Placebo + BCG & $32(32 / 1003)$ & $37(18 / 490)$ & $27(14 / 512)$ \\
\hline Mortality rate ratio $(95 \% \mathrm{Cl})$ & $1.84(1.19$ to 2.85$)$ & 1.53 (0.84 to 2.79$)$ & $2.23(1.18$ to 4.24$)$ \\
\hline \multicolumn{4}{|l|}{ Dry season } \\
\hline Vitamin A + BCG & $39(34 / 882)$ & $31(14 / 448)$ & $46(20 / 433)$ \\
\hline Placebo + BCG & $61(54 / 881)$ & $69(32 / 462)$ & $53(22 / 418)$ \\
\hline Mortality rate ratio $(95 \% \mathrm{Cl})$ & $0.63(0.41$ to 0.97$)$ & $0.45(0.24$ to 0.84$)$ & $0.88(0.48$ to 1.61$)$ \\
\hline Homogeneity of effects & $P=0.0006$ & $P=0.0058$ & $P=0.0382$ \\
\hline
\end{tabular}

The effect of vitamin A supplementation on mortality did not differ by maternal education overall. In boys of mothers with no education the mortality rate ratio was 0.42 (0.17 to 1.02$)$ compared with $1.04(0.61$ to 1.78$)$ in boys of mothers with schooling ( $\mathrm{P}$ for interaction=0.09), whereas no difference existed in girls. The effect of vitamin A supplementation did not differ significantly according to maternal arm circumference or children's anthropometrics at enrolment. However, in infants with a ponderal index in the lowest quarter $\left(<24.7 \mathrm{~kg} / \mathrm{m}^{3}\right)$, the mortality rate ratio was $0.69(0.34$ to 1.38) compared with 1.35 (0.86 to 2.13 ) in other children ( $\mathrm{P}$ for interaction=0.11). This was mainly caused by a significant negative effect of vitamin A supplementation among girls with a normal or high ponderal index-2.14 (1.06 to 4.29) compared with $0.80(0.30$ to 2.16$)$ in girls with a low ponderal index $(\mathrm{P}$ for interaction $=0.11$ ).

\section{Season}

We also did an explorative analysis of the effect of vitamin A supplementation according to the season of administration. As in many previous studies from the study area, we considered the rainy season to last from June to November, even though the rain may have stopped in early October. The incidence of malaria is usually particularly high in October and November. We found a strong interaction between vitamin A and season of distribution. Vitamin A supplementation administered in the dry season was associated with a mortality rate ratio of 0.63 (0.41 to 0.97$)$, whereas vitamin A supplementation administered in the rainy season was associated with a mortality rate ratio of 1.84 (1.19 to 2.85) (table 2). The negative effect of receiving supplement in the rainy season was evident in boys as well as girls. As a consequence, vitamin A was associated with a significantly increased mortality in girls supplemented in the rainy season (mortality rate ratio 2.23 (1.18 to 4.24$) ; \mathrm{P}=0.01$ ) but a significantly beneficial effect among boys supplemented in the dry season (0.45 (0.24 to 0.84$) ; \mathrm{P}=0.01)$.

\section{Causes of death}

In $21(12 \%)$ of the 174 deaths, a verbal autopsy was not done because the family had moved or refused to participate. Among the remaining 153 deaths, several had more than one probable cause of death. Main causes of death were septicaemia, malaria, diarrhoea, and respiratory infections. On the basis of the observations of sex differential and season differential effects of vitamin A supplementation on mortality, we did exploratory analyses of the effect of vitamin A supplementation by sex and season (table 3 ). The negative effect of vitamin A supplementation administered in the rainy season was apparent for deaths from malaria, diarrhoea, and respiratory infections but not for deaths from septicaemia. The sex differential effect was most apparent for diarrhoea; vitamin A supplementation was associated with significantly more deaths from diarrhoea among girls, particularly if they received supplement in the rainy season. A total of 
Table $3 \mid$ Mortality rate ratios ( $95 \%$ confidence intervals) for vitamin A supplementation versus placebo for different causes of death by season of supplementation and sex

\begin{tabular}{|c|c|c|c|c|}
\hline \multirow{2}{*}{$\begin{array}{l}\text { Cause of death, by season of } \\
\text { supplementation }\end{array}$} & \multirow[b]{2}{*}{ No of deaths* } & \multicolumn{3}{|c|}{ Vitamin $\mathrm{A} /$ placebo } \\
\hline & & All & Boys & Girls \\
\hline Malaria: & 32 & $0.9(0.5$ to 1.9$)$ & $0.5(0.1$ to 1.5$)$ & 1.4 (0.6 to 3.6$)$ \\
\hline Rainy & 12 & $2.2(0.7$ to 7$)$ & $2.2(0.2$ to 23.8$)$ & 2.2 (0.6 to 8.9$)$ \\
\hline Dry & 20 & $0.5(0.2$ to 1.4$)$ & 0.3 (0.1 to 1.2$)$ & $1.0(0.3$ to 3.3$)$ \\
\hline Septicaemia: & 26 & 1.2 (0.6 to 2.6$)$ & 1.8 (0.6 to 5.5$)$ & 0.7 (0.2 to 2.3$)$ \\
\hline Rainy & 15 & $1.2(0.4$ to 3.3$)$ & 2.3 (0.6 to 9.1) & 0.3 (0.0 to 2.5$)$ \\
\hline Dry & 11 & $1.2(0.4$ to 3.9$)$ & 1.0 (0.1 to 7.3$)$ & $1.3(0.3$ to 5.8$)$ \\
\hline Diarrhoea: & 33 & $1.6(0.8$ to 3.2$)$ & 0.7 (0.3 to 2.0$)$ & $3.7(1.2$ to 11.1$) \dagger$ \\
\hline Rainy & 20 & 2.6 (1.0 to 6.7$)$ & $1.1(0.3$ to 4.3$)$ & $5.6(1.2$ to 25.5$)$ \\
\hline Dry & 13 & $0.9(0.3$ to 2.6$)$ & $0.4(0.1$ to 2.1$)$ & 1.9 (0.3 to 10.6$)$ \\
\hline Respiratory infection: & 25 & $1.1(0.5$ to 2.5$)$ & 1.1 (0.3 to 3.6$)$ & 1.2 (0.4 to 3.3 ) \\
\hline Rainy & 12 & $2.2(0.7$ to 7.3$)$ & $1.1(0.2$ to 7.6$)$ & $3.3(0.7$ to 16.6$)$ \\
\hline Dry & 13 & $0.6(0.2$ to 1.9$)$ & $1.0(0.2$ to 5.1$)$ & 0.4 (0.1 to 2.0$)$ \\
\hline Measles & 16 & $0.8(0.3$ to 2.2$)$ & 0.5 (0.1 to 2.1$)$ & $1.4(0.3$ to 6.3$)$ \\
\hline Meningitis & 4 & 0.3 (0.0 to 3.3 ) & NA & NA \\
\hline Acute febrile syndrome & 8 & $1.0(0.3$ to 4.2$)$ & 0.4 (0.0 to 3.4$)$ & 3.1 (0.3 to 39.6$)$ \\
\hline Other infection & 5 & 0.7 (0.1 to 4.1) & NA & NA \\
\hline $\begin{array}{l}\text { Maybe non-infectious } \\
\text { underlying condition } \ddagger\end{array}$ & 14 & $1.4(0.5$ to 4.0$)$ & $1.0(0.3$ to 4.1$)$ & 2.1 (0.4 to 11.3 ) \\
\hline Fever combined & 69 & $1.0(0.6$ to 1.6$)$ & 0.9 (0.4 to 1.7$)$ & 1.2 (0.6 to 2.2 ) \\
\hline rainy & 30 & $1.6(0.8$ to 3.4$)$ & 2.1 (0.7 to 6.1$)$ & 1.3 (0.5 to 3.5$)$ \\
\hline dry & 39 & $0.7(0.4$ to 1.3$)$ & $0.4(0.1$ to 1.1$)$ & 1.1 (0.5 to 2.5$)$ \\
\hline
\end{tabular}

$\mathrm{NA}=$ not applicable.

*Total denominator was 3677.8 person years: 1915.4 in rainy season and 1762.4 in dry season.

$\dagger$ Significant difference between effects of vitamin A supplementation in boys and girls, $P=0.03$.

$\ddagger$ These children were judged to have died from conditions such as sudden infant death syndrome $(n=2)$,

hydrocephalus or increased intracranial pressure $(n=3)$, and acute bleeding $(n=5)$.

94 children were exposed to tuberculosis. Two $(2 \%)$ deaths occurred among these children, both in the placebo group; one of them refused verbal autopsy but was said to have died from diarrhoea and vomiting, and the other died of pneumonia.

\section{DISCUSSION}

\section{Principal findings}

In this trial, we were unable to show an important effect of vitamin A supplementation on overall mortality, but the effect of supplementation tended to be beneficial for boys but harmful for girls. In retrospective analyses, we saw a strong interaction between vitamin A supplementation and season; vitamin A supplementation was beneficial when provided in the dry season but detrimental when provided in the rainy season.

\section{Strengths and weaknesses}

Strengths of the study include the demographic surveillance system that has been functioning for many years; thus, for an African setting we have been able to keep track of a very large percentage of the children enrolled at the hospital and at the health centres. All eligible children were enrolled in the study. The doctors and the assistants at the project have extensive experience in this type of study, and we believe it has been conducted rigorously.

Limitations include the fact that the mortality was much lower (47/1000 person years) than anticipated
(70/1000 person years), probably in part because we offered free consultations and essential drugs during the first year of life for eligible children. The randomisation method was unconventional but was carried out under careful supervision. We preferred it to other methods such as computer generated treatment allocation, as it made it clear to the mothers that they were participating in a randomised trial. Furthermore, interviews with mothers and assistants indicated that the blinding was successful, and no differences in baseline characteristics existed between treatment groups. We do not believe that these weaknesses have influenced the results, but the low mortality obviously decreased our power to estimate the effect of vitamin A supplementation, and the confidence interval of the overall estimate still leaves room for clinically important effects.

One could speculate that the oral vitamin A supplement was not well absorbed in neonates, as we found no differences in vitamin A concentrations at 6 weeks of age ${ }^{10}$ However, given the facts that neonatal oral vitamin A supplementation affected overall mortality in previous trials and sex differential mortality in this as well as previous trials, ${ }^{34}$ and that a previous study showed increased plasma retinol concentrations after neonatal oral vitamin A supplementation, ${ }^{17}$ we believe that the vitamin A was absorbed. In this trial, we also found vitamin A supplementation to be associated with higher concentrations of retinol binding protein at 6 weeks of age among boys of mothers with no formal education. ${ }^{10}$

\section{Possible explanations of contrasting results}

When we started our trial, two similar trials from Asia had reported beneficial effects of vitamin A supplementation at birth. ${ }^{34}$ While our trial was ongoing, a trial from Zimbabwe supplementing HIV negative women and their offspring in a two by two factorial design could not reproduce a beneficial effect of vitamin A supplementation at birth; the effect of neonatal vitamin A supplementation during the first 12 months of life was $1.18 .{ }^{18}$ Recently, the Zimbabwe trial reported that among infants of HIV positive mothers, vitamin A

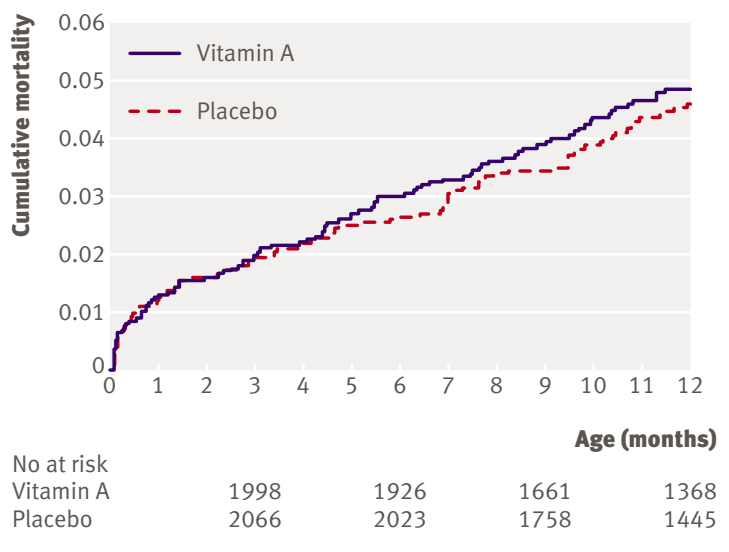

Fig 2 Kaplan-Meier estimates of death in vitamin A and placebo recipients 
supplementation had differential effects in infants who were polymerase chain reaction negative for HIV at birth but became positive at 6 weeks of age and children who remained negative six weeks after delivery. ${ }^{19}$ The overall effect of neonatal vitamin A supplementation in the combined population was 1.16 (0.98 to 1.38$).{ }^{19}$ The Zimbabwe trial was done in an area that is not endemic for malaria, and this was speculated to be the reason for the apparent lack of a beneficial effect. However, we found no effect of vitamin A supplementation in a malaria endemic area and no effect of vitamin A supplementation against deaths from malaria.

The trial populations were all large $(n=2067$ $14110^{31819}$ ) and to our knowledge conducted rigorously. The conflicting results could be due to an underlying difference in degree of vitamin A deficiency. In Indonesia, the maternal retinol concentrations were comparable to those of American women. ${ }^{3}$ In India, $6 \%$ of the mothers had night blindness and the degree of underlying vitamin A supplementation was probably high. ${ }^{4}$ In Zimbabwe, less than $1 \%$ of the mothers were deficient $(<0.7 \mu \mathrm{mol} / 1$ retinol binding protein). ${ }^{18}$ In Bissau, less than $1 \%$ of the mothers had low retinol binding protein. ${ }^{10}$ As the direct measures of vitamin A status varied between the studies, no firm conclusions can be reached, but nothing indicates that differences in vitamin A status alone can account for the apparent beneficial effect in Indonesia and India but not in Zimbabwe or Guinea-Bissau.

Maternal education is an important socioeconomic indicator, and vitamin A status may differ by maternal education. ${ }^{20}$ In our trial, educated mothers had significantly higher retinol binding protein than mothers with no education, ${ }^{10}$ and vitamin A supplementation seemed to have a more beneficial effect in boys of mothers with no education. The mean maternal education was 9.5 years in Indonesia, ${ }^{3}$ and $83 \%$ had eight or more years of education in Zimbabwe, ${ }^{18}$ whereas more than $40 \%$ of the mothers in India and more than $28 \%$ of the mothers in Guinea-Bissau had no education. ${ }^{4}$ Hence, difference in maternal education seems unlikely to explain the conflicting results.

The percentage of low birthweight children differed considerably between studies. Low birthweight children are at greatest risk of vitamin A deficiency. ${ }^{21}$ Our results indicated that girls with a high ponderal index benefited least from vitamin A supplementation. Our study did not include low birthweight children, whereas the studies in Indonesia, India, and Zimbabwe had $10 \%, 31 \%$ and $12 \%$ low birthweight children respectively. ${ }^{3418}$ Hence, differences in birth weight do not seem to explain the differences either.

\section{Sex differences}

The two Asian studies reported a more beneficial effect in boys, a finding that we could replicate in our study, although it was not significant at the 5\% level. However, unlike the Asian studies we found a tendency for a negative effect in girls. The Zimbabwean study did not report data by sex. The very first vitamin A supplementation study by Sommer et al found a more

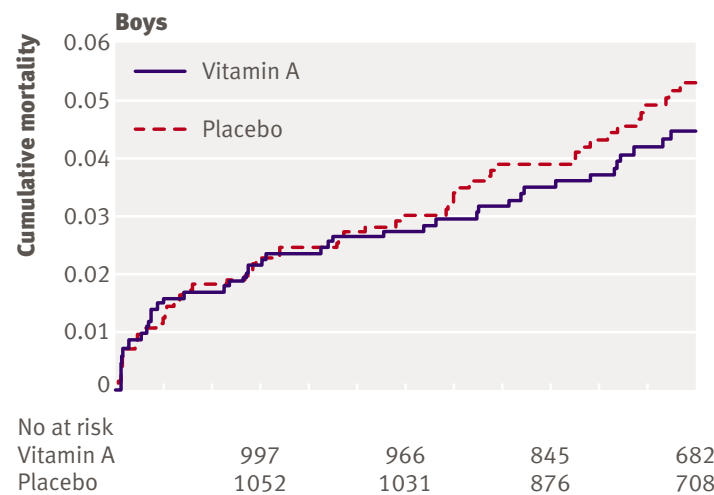

beneficial effect in infant boys than girls. ${ }^{22}$ We have previously reported that boys had a stronger antibody response from measles vaccine administered with vitamin A supplementation, ${ }^{23}$ and girls in contrast to boys benefited from receiving lower doses of vitamin A supplementation than the ones currently recommended. ${ }^{5}$ The reason for these differences between the sexes is unknown but potentially very important for designing the optimal vitamin A policy.

\section{Seasonal effects}

An explorative analysis revealed a strong interaction with season of supplementation, resulting in a significantly beneficial effect of vitamin A supplementation if given to boys during the dry season but a significantly negative effect if given to girls during the rainy season. The analyses of causes of deaths by season revealed that vitamin A supplementation administered in the rainy season tended to be associated with increased mortality from malaria, diarrhoea, and respiratory infections. Hence, the negative effect of vitamin A supplementation in the rainy season was not due to a negative effect on one particular type of disease. Several studies have reported that the growth response to vitamin A supplementation depended on season. ${ }^{24-26}$ One trial from Ghana reported no differential effect of vitamin A supplementation distributed at three different times of the year $(\mathrm{P}=0.16),{ }^{27}$ but the effects of number of doses and time of the year could not be separated. The effect of 


\section{WHAT IS ALREADY KNOWN ON THIS TOPIC}

Vitamin A supplementation at birth has been associated with decreased mortality in Asia

\section{WHAT THIS STUDY ADDS}

No important beneficial effect of vitamin A supplementation was found in an African setting

Vitamin A supplementation may benefit boys more than girls

Vitamin A supplementation interacted strongly with season, being beneficial during the dry season but harmful during the rainy season

vitamin A supplementation administered in the dry season in Bissau was very similar to effects obtained in the two Asian studies. None of the other studies of neonatal vitamin A supplementation reported effects by season. If health interventions such as vitamin $\mathrm{A}$ supplementation have differential seasonal effects, this would have major consequences for public health practice in low income countries-for instance, when to run vitamin A supplementation campaigns.

\section{Biological mechanisms}

We have no well documented explanation of why vitamin A supplementation should have sex differential and season differential effects. One could speculate that underlying differences in the prevalence of vitamin A deficiency between the sexes and between seasons might explain the results. Indeed, the indications are that boys are born with lower vitamin A concentrations. ${ }^{2829}$ However, in our population boys were no more vitamin A deficient than girls at 6 weeks, and at 4 months they had higher retinol binding protein concentrations than girls. ${ }^{10}$ If anything, children were less vitamin A deficient during the dry season. ${ }^{10}$

In immunological studies, vitamin A has mostly been shown to enhance $\mathrm{T}$ helper (Th) 2 type responses..$^{30}$ To our knowledge, no study has systematically investigated the Th1/Th2 balance in infant boys and girls, although the indications are that boys may have a more pronounced Th1 profile than girls as they have a stronger delayed hypersensitivity response. ${ }^{13}$ Conversely, girls may have a stronger Th2 profile as they usually mount a higher antibody response than boys. ${ }^{23}$ Hence, we might speculate that the sex differential effect is due to vitamin A supplementation inducing a more balanced and beneficial Th1/Th2 profile in boys but unnecessary or excessive Th2 deviation in girls.

Treatment with vitamin A has been shown to have disease specific effects; for instance, vitamin A treatment is beneficial against measles but may enhance the clinical symptoms during respiratory infections. ${ }^{30}$ The seasonal differences could be due to seasonal differences in the prevalence of certain disease types against which vitamin A supplementation is particularly beneficial or harmful. Our data suggest that the negative effect of vitamin A supplementation administered in the rainy season was seen for several of the major causes of death. However, we did not have very advanced diagnostic tools and hence not very specific diagnoses. We could also speculate that the season differential effect was due to differences in the state of the immune system during different seasons. In the neighbouring country the Gambia, seasonal difference in the antibody response to several different vaccines has been noted. ${ }^{31}$

In conclusion, more studies focusing on the immunological effects of vitamin A supplementation and its interactions with sex and seasonal differences in immune function are clearly needed to elucidate the potential biological mechanisms.

\section{Overall evidence}

A recent paper estimated that $42 \%$ of children aged 0-59 months in sub-Saharan Africa are at risk of vitamin A deficiency and that programme action for control of vitamin A deficiency can bring about a potential 25\% reduction in mortality in this age group. ${ }^{32} \mathrm{We}$ did this study believing that vitamin A supplementation would contribute to further reductions in mortality in our African setting. This did not happen. As indicated by the effect in the dry season, particularly in boys, vitamin A supplementation may indeed have a beneficial effect in some situations. However, it apparently also interacted with other, as yet unknown, environmental factors to produce a negative effect on survival in some situations.

Four trials of vitamin A supplementation at birth have now been published. Two Asian studies found a beneficial effect of vitamin A supplementation ${ }^{34}$; two African studies found no overall benefit and possibly harmful effects in girls (our study) and in HIV negative children born of HIV positive mothers. ${ }^{19}$ The three trials that reported data by sex (including ours) found a more beneficial effect in boys. ${ }^{34}$ A meta-analysis of the four trials of supplementation at birth shows significant heterogeneity in the mortality effect of vitamin A supplementation between the trials $(\mathrm{P}=0.003)$. Hence, some unidentified factor(s) seems to exist that explains whether vitamin A supplementation has a beneficial effect, no effect, or a negative effect.

On the basis of published studies and preliminary results from an as yet unpublished study in Bangladesh showing a beneficial effect of vitamin A supplementation at birth, ${ }^{33}$ recommendation of neonatal vitamin $\mathrm{A}$ supplementation at birth in Asia, but not in Africa, has recently been suggested. ${ }^{3435}$ However, before moving to policy we need to resolve the contradictions to identify the situations in which vitamin A supplementation is beneficial or harmful and to clarify the underlying mechanisms. Only by doing so can we optimise the use of vitamin A supplementation in low income countries. As long as the causes of the contrasting results are not identified, the possibility exists that vitamin A supplementation at birth might be harmful for some groups. Given the current evidence, a global or regional recommendation of 50000 IU vitamin A supplementation at birth is unwarranted.

Contributors: CSB was the chief investigator and is the guarantor. CSB, ARoth, MY, HW, ARodrigues, and PA designed the study. CSB, ARoth, and $P A$ initiated the study. BRD, EN, ABF, and IML were responsible for the 
recruitment and follow-up of participants. CSB was responsible for the statistical analysis and wrote the first draft of the paper. All authors contributed to and approved the final version of the paper. Funding: The study was funded by the EU (ICA4-CT-2002-10053), the Danish Medical Research Council, University of Copenhagen, March of Dimes, and the Ville Heise Foundation. The Bandim Health Project received support from DANIDA and the Danish National Research Foundation. PA holds a research professorship grant from the Novo Nordisk Foundation. The sponsors had no role in the study design, data collection, data analysis, data interpretation, or the writing of the report Competing interests: None declared.

Ethical approval: Ministry of Health in Guinea-Bissau; Danish Central Ethical Committee (624-02-0010).

Provenance and peer review: Not commissioned; externally peer reviewed.

1 Fawzi WW, Chalmers TC, Herrera MG, Mosteller F. Vitamin A supplementation and child mortality: a meta-analysis. JAMA 1993;269:898-903.

2 Benn CS, Balé C, Sommerfelt H, Friis H, Aaby P. Vitamin A supplementation and childhood mortality: amplification of the nonspecific effects of vaccines? Int J Epidemiol 2003;32:822-8.

3 Humphrey JH, Agoestina T, Wu L, Usman A, Nurachim M, Subardja D, et al. Impact of neonatal vitamin A supplementation on infant morbidity and mortality. J Pediatr 1996;128:489-96.

4 Rahmathullah L, Tielsch JM, Thulasiraj RD, Katz J, Coles C, Devi S, et al. Impact of supplementing newborn infants with vitamin A on early infant mortality: community based randomised trial in southern India. BMJ 2003;327:254.

5 Benn CS, Martins C, Rodrigues A, Jensen H, Lisse IM, Aaby P. Randomised study of the impact of different doses of vitamin $A$ on childhood morbidity and mortality. BMJ 2005;331:1428-32.

6 Benn CS, Fisker AB, Diness BR, Aaby P. Sex-differential effect of neonatal vitamin A supplementation on mortality? I Infect Dis 2006;194:721.

7 Benn CS, Fisker AB, Jørgensen MJ, Aaby P. Why worry: vitamin A with DTP vaccine? Vaccine 2007;25:777-9.

8 Adjorlolo-Johnson G, De Cock KM, Ekpini E, Vetter KM, Sibailly T, Brattegaard K, et al. Prospective comparison of mother-to-childtransmission of HIV-1 and HIV-2 in Abidhan, Ivory Coast. JAMA 1994:272:462-6.

9 Unicef. Vitamin and mineral deficiency. New York: Unicef, 2004 (www.unicef.org/videoaudio/PDFs/africa_dar.pdf).

10 Fisker AB, Lisse IM, Aaby P, Erhardt J, Rodrigues A, Bibby BM, et al. Impact of neonatal vitamin $A$ supplementation with $B C G$ vaccine on vitamin A status at 6 weeks and 4 months of age. Am / Clin Nutr 2007;86:1032-9.

11 WHO/Unicef/IVACG Taskforce. Vitamin A supplements: a guide to their use in the treatment and prevention of vitamin A deficiency and xerophthalmia. 2nd ed. Geneva: WHO, 1997.

12 Nante JE, Diness BR, Ravn H, Roth A, Aaby P, Benn CS. No adverse events after simultaneous administration of 50,000 IU vitamin $A$ and Bacille Calmette-Guerin vaccination to normal-birth-weight newborns in Guinea-Bissau. Eur / Clin Nutr 2007, doi: 10.1038/sj.ejcn.1602796.

13 Diness BR, Fisker AB, Roth A, Yazdanbakhsh M, Sartono E, Whittle H, et al. The effect of high-dose vitamin A supplementation on the immune response to Bacille Calmette-Guerin vaccination. Am J Clin Nutr 2007;86:1152-9.

14 Indepth Network. Standard verbal autopsy questionnaire. 2003. www.indepth-network.org/core_documents/downloads.htm.

15 Anker M, Black RE, Coldham C, Kalter HD, Quigley MA, Ross D, et al. A standard verbal autopsy method for investigating causes of death in infants and children. Geneva: WHO,

1999 (available at www.who.int/csr/resources/publications/ surveillance/whocdscsrisr994.pdf).

16 Cox DR, Oakes D. Analysis of survival data. London: Chapman \& Hall, 1984.
17 Coutsoudis A, Adhikari M, Pillay K, Coovadia HM. Absorption of highdose enteral vitamin A in low-birth-weight neonates. S Afr Med I 1996;86:1337-9.

18 Malaba LC, lliff PJ, Nathoo KJ, Marinda E, Moulton LH, Zijenah LS, et al. Effect of postpartum maternal or neonatal vitamin A supplementation on infant mortality among infants born to HIV-negative mothers in Zimbabwe. Am J Clin Nutr 2005;81:454-60.

19 Humphrey JH, lliff PJ, Marinda ET, Mutasa K, Moulton LH, Chidawanyika $\mathrm{H}$, et al. Effects of a single large dose of vitamin $\mathrm{A}$, given during the postpartum period to HIV-positive women and their infants, on child HIV infection, HIV-free survival, and mortality. J Infect Dis 2006;193:860-71.

20 Kjolhede CL, Stallings RY, Dibley MJ, Sadjimin T, Dawiesah S, Padmawati S. Serum retinol levels among preschool children in Central Java: demographic and socioeconomic determinants. Int J Epidemiol 1995;24:399-403.

21 Rondo PH, Abbott R, Tomkins AM. Vitamin A and neonatal anthropometry. J Trop Pediatr 2001;47:307-10.

22 Sommer A, Tarwotjo I, Djunaedi E, West KP Jr, Loeden AA, Tilden R, et al. Impact of vitamin A supplementation on childhood mortality: a randomised controlled community trial. Lancet 1986;:1169-73.

23 Benn CS, Aaby P, Balé C, Olsen J, Michaelsen KF, George E, et al. Randomised trial of effect of vitamin A supplementation on antibody response to measles vaccine in Guinea-Bissau, West Africa. Lancet 1997;350:101-5.

24 Hadi H, Dibley MJ, West KP Jr. Complex interactions with infection and diet may explain seasonal growth responses to vitamin $A$ in preschool aged Indonesian children. Eur J Clin Nutr 2004;58:990-9.

25 Bahl R, Bhandari N, Taneja S, Bhan MK. The impact of vitamin A supplementation on physical growth of children is dependent on season. Eur J Clin Nutr 1997;51:26-9.

26 West KP, LeClerq SC, Shrestha SR, Wu LS, Pradhan EK, Kathry SK, et al. Effects of vitamin A on growth of vitamin A-deficient children: field studies in Nepal. J Nutr 1997;127:1957-65.

27 Ross DA, Kirkwood BR, Binka FN, Arthur P, Dollimore N, Morris SS, et al. Child morbidity and mortality following vitamin $A$ supplementation in Ghana: time since dosing, number of doses and time of the year. Am J Publ Health 1995;85:1246-51.

28 Yassai MB, Malek F. Newborns vitamin A in relation to sex and birth weight. J Trop Pediatr 1989;35:247-9.

29 Tolba AM, Hewedy FM, al-Senaidy AM, al-Othman AA. Neonates' vitamin A status in relation to birth weight, gestational age, and sex. J Trop Pediatr 1998;44:174-7.

30 Villamor E, Fawzi WW. Effects of vitamin A supplementation on immune responses and correlation with clinical outcomes. Clin Microbiol Rev 2005;18:446-64.

31 Moore SE, Collinson AC, Fulford Al, Jalil F, Siegrist CA, Goldblatt D, et al. Effect of month of vaccine administration on antibody responses in the Gambia and Pakistan. Trop Med Int Health 2006;11:1529-41.

32 Aguayo VM, Baker SK. Vitamin A deficiency and child survival in subSaharan Africa: a reappraisal of challenges and opportunities. Food Nutr Bull 2005;26:348-55.

33 Klemm R, Labrique A, Christian P, Rashid M, Shamim AA, Wu L, et al. Efficacy of newborn vitamin A supplementation in reducing infant mortality in rural Bangladesh: the JIVITA-2 trial. Abstract presented at the 1st Micronutrient Forum Meeting in Istanbul, Turkey, April 2007. www.micronutrientforum.org/Meeting2007/MN\%20Forum\% 20Program\%20Part\%20ll_Abstracts.pdf (abstract T115).

34 West Jr K, Sommer A. Newborn vitamin A dosing: policy implications for Southern Asia and Africa. Abstract presented at the 1st Micronutrient Forum Meeting in Istanbul, Turkey, April 2007. www. micronutrientforum.org/Meeting2007/MN\%20Forum\%20Program \%20Part\%20ll_Abstracts.pdf (abstract T116).

35 Bhutta ZA, Ahmed T, Black RE, Cousens S, Dewey K, Giugliani E, et al. What works? Interventions for maternal and child undernutrition and survival. Lancet 2008;371:417-40.

Accepted: 19 March 2008 